


\title{
ANÁLISIS DE LA EFICIENCIA DE LOS PROCESOS DE REESTRUCTURACIÓN DE PLANTILLA EN LAS PRINCIPALES ENTIDADES FINANCIERAS ESPAÑOLAS
}

\author{
Autor: Isabel Lázaro Aguilera ${ }^{1}$ \\ Profesora colaboradora de la Universidad CEU San Pablo
}

\section{Resumen}

Una respuesta generalizada por parte de las empresas en tiempos de crisis económica ha sido reducir el número de empleados a través de los procesos de reestructuración de plantilla. Este trabajo analiza el efecto que estos procesos, centrándonos en el caso concreto de los despidos, han tenido sobre los niveles de rentabilidad, eficiencia y productividad, en las cinco entidades principales del sistema financiero español. Para ello, el estudio utiliza una muestra de las cinco empresas del sector financiero que cotizan en el IBEX 35 abarcando un período temporal de diez años (2003-2012).

\footnotetext{
${ }^{1}$ mariaisabel.lazaroaguilera@ceu.es
} 
Palabras clave: Reestructuración de plantilla; Downsizing; Despidos; Eficiencia; Rentabilidad; Productividad.

\title{
Measuring the efficiency of restructuring in the main spanish financial ins- titutions.
}

\begin{abstract}
During a recession, many firms favour a strategy of reducing the number of employees by restructuring their workforce. Focusing on the particular case of redundancy, this study will analyze the effect that downsizing has had on levels of profitability, efficiency and productivity, on the top five entities of the Spanish banking system/financial sector. With this in mind, the study uses the example of five organizations in the financial sector that are all listed on the IBEX 35 over a 10 year time span (2003-2012).
\end{abstract}

Key words: Workforce restructuring; Downsizing; Layoffs; efficiency; profitability; productivity.

\section{INTRODUCCIÓN}

En los tiempos que corren en los que una gran mayoría de empresas, tanto a nivel nacional como internacional, tienen que afrontar una situación de crisis global, es cuando los empresarios tienden a solucionar sus problemas de viabilidad y es en este momento cuando las empresas comienzan a plantearse las reestructuraciones de plantilla y en concreto una de sus modalidades, el downsizing ${ }^{2}$. De esta manera las empresas consiguen reajustar sus costes laborales para intentar mejorar la competitividad de su organización y conseguir, así una garantía de supervivencia en el futuro. De ahí que las organizaciones hayan comenzado a comprender que la reestructuración es una parte más de la gestión empresarial y que es necesario hacer, en la medida de lo posible, una gestión anticipada que intente poner los medios e instrumentos para que sus efectos colaterales sean los mínimos posibles.

\footnotetext{
2 Podemos definir el downsizing como la decisión de reducir el tamaño de la plantilla de la empresa como resultado de un proceso de cambio estratégico (Cascio,1993).
} 
En ocasiones, los procesos de reestructuración de plantilla son difícilmente evitables puesto que forman una parte intrínseca de la vida de las organizaciones en las que en algún momento, por circunstancias externas del mercado, es necesario realizar estos ajustes para asegurar la supervivencia de las mismas. A la hora de llevar a cabo estos procesos, la decisión a ejecutar debe basarse, ante todo, en un análisis profundo de su rentabilidad operativa y humana. Por ello, la mayor dificultad de estos procesos de reestructuración se centra siempre en la valoración del coste humano puesto que dicho coste resulta difícilmente cuantificable.

Dentro de la literatura académica acerca de los efectos o las consecuencias de las reestructuraciones de plantillas podemos encontrar tres grupos de trabajos en función del tipo de efectos que analizan: 1) enfoque económico-financiero, 2) enfoque psicosocial y de recursos humanos, y 3 ) enfoque organizativo. Desde el enfoque económico-financiero se analizan las consecuencias de estas estrategias sobre los resultados contables y en la capitalización bursátil de las empresas que han llevado a cabo estas técnicas (Worrell et.al, 1991; Mentzer, 1996; Cascio et.al, 1997; Wayham y Werner, 2000; Nixon et al., 2004; De Meuse et.al, 2004). A grandes rasgos, se puede decir que esta literatura no es concluyente sobre si realmente se obtienen los resultados esperados. El interés básico del enfoque psicosocial y de recursos humanos se centra en las consecuencias sobre la moral y la motivación en los empleados (Brockner et.al, 1992; Nantaporn y Kleiner, 2003). Estos trabajos sí son concluyentes puesto que muestran con un nivel alto de significación los resultados negativos que genera la reducción de plantilla. Finalmente, el enfoque organizativo analiza sus efectos en las estructuras organizativas (DeWitt, 1993; McKinley et.al, 1998).

Dados los objetivos del presente trabajo, el estudio se centrará en las consecuencias económico-financieras como base conceptual de partida para llevar a cabo el análisis empírico.

A pesar de que estas prácticas se han generalizado a nivel mundial en las grandes empresas (Cascio, 1993; Cameron et.al, 1993; DeWitt, 1993; Hitt et.al, 1994; Markides, 1995; Bruton et.al, 1996; Mentzer, 1996; Morris et. at., 1999; Filatotchev et.al, 2000; Ahmadjian y Robinson, 2001; Hillier, 2007; Pfeifer, 2007) y de que, también, han sido adoptadas frecuentemente por las mayores empresas españolas (Requejo 1996; Casillas y Barroso, 1998; Suárez, 1999; Sánchez y Suárez, 2005; Claver y Fernández, 2006; Fernández, 2006; Odette et.al, 2007; Muñóz y Sánchez, 2010, 2011; Suarez y Vicente, 2009), existen todavía muchos interrogantes sobre este tema puesto que no se ha podido comprobar efectivamente que los efectos de estas técnicas sean los perseguidos inicialmente.

Una de las cuestiones más debatidas dentro de la literatura con estudios empíricos sobre el downsizing es la confirmación o, en su defecto, el rechazo de la hipótesis sobre la eficiencia económica de estas técnicas, esto es, si las empresas que hacen reestructuración de plantilla consiguen mejorar sus resultados organizativos. A este respecto, la evidencia disponible está lejos de ser concluyente ya que existen tra- 
bajos que lo asocian, por un lado, a mejores resultados (Cascio, 1993; De Meuse et al., 2004; Espahbodi et.al, 2000; Cappelli, 2000) y, por otro, a efectos negativos (Budros, 1999; Morris et.al, 1999; Nixon et.al, 2004; Hillier, 2007).

El principal motivo por el que se encuentran resultados contradictorios radica en el hecho de que cada investigación se realiza con una muestra diferente (país, años, sector empresarial, variables). Además de esto debe tenerse presente que cada país tiene su propia regulación referente al mercado laboral, hecho que afecta de manera directa a los tipos de contratos realizados en las empresas y por ende a las técnicas de implementación de downsizing que se emplean y al tamaño de estas reducciones.

En relación con lo anterior se sabe que, tradicionalmente, se ha supuesto que las organizaciones deciden reducir voluntariamente su tamaño con tres finalidades:

- Conseguir mejoras en la rentabilidad,

- recortar costes y,

- lograr incrementos en su productividad.

Sin embargo, los resultados empíricos aportados por los trabajos de investigación que se han realizado sobre el tema, no lo han corroborado. No obstante, todos los autores coinciden en que las consecuencias que tienen estas técnicas sobre el personal son negativas. Este hecho nos lleva a cuestionarnos si las reestructuraciones de plantilla y en concreto, la modalidad del downsizing es un buen método porque si las consecuencias son nefastas sobre los empleados y, además, no existe evidencia de eficiencia, se plantean serias dudas sobre si estas técnicas de reestructuración resultan beneficiosas de alguna manera para las organizaciones.

Es importante tener en cuenta cuales han sido los distintos motivos que han propiciado estas prácticas de reducción de plantilla puesto que éstos pueden conducir a distintos resultados para la estrategia de downsizing. Existen diferentes políticas para llevar a cabo los procesos de reestructuración de plantilla, puesto que los puestos de trabajo que se eliminan no se traducen únicamente en despidos, sino que también se concentran en recolocaciones de empleados, prejubilaciones y bajas incentivadas. La mayoría de los estudios se centran en la modalidad de los despidos. Hay que tener en cuenta que el contexto legislativo en el que están inmersas las organizaciones puede favorecer la elección de un tipo de prácticas o de otras (Dahl y Nesheim, 1998), pero también el peso que juegan otro tipo de factores organizativos y económicos promotores de estas técnicas de downsizing pueden diferir entre estas opciones.

En este trabajo de investigación se pretende analizar el efecto que los procesos de reestructuración de plantilla, centrándonos en el caso concreto de la reducción de plantilla han tenido sobre los niveles de rentabilidad, eficiencia y productividad en las entidades del sistema financiero español a raíz de la crisis económica en la que se encuentra sumergido nuestro país. Con este estudio se pretende contribuir a la escasa evidencia empírica sobre las consecuencias que 
los las estrategia de downsizing tienen sobre las principales entidades financieras españolas, lo que nos permitirá analizar si existen diferencias o similitudes respecto a otros contextos en cuanto a los efectos en los resultados de estas técnicas.

El objetivo que se plantea con este trabajo radica en la necesidad de analizar si las técnicas de reestructuración empleadas en las entidades que forman parte del sistema financiero español ${ }^{3}$, en concreto los bancos que cotizan en el IBEX 35, han logrado los resultados esperados en las ratios de rentabilidad de las empresas y, por tanto, consiguen mejorar la eficiencia.

Mediante la presente investigación se pretende evaluar la eficiencia de las entidades financieras que realizaron estas técnicas a partir de los resultados económico-financieros obtenidos en el estudio.

\section{MARCO CONCEPTUAL}

\subsection{Concepto de reestructuración de plantilla}

\subsubsection{Aproximación al fenómeno}

Después de la segunda guerra mundial y hasta los años 80 , la tendencia general de las grandes empresas fue a crecer tanto en sus negocios originales como diversificándose a otros nuevos (Chandler, 1990 y Rumelt, 1974, citado en Suarez, 1999). Sin embargo, existe ya una importante evidencia de que en la actualidad la tendencia es más bien a la inversa: muchas de esas grandes empresas están ahora inmersas en una ola masiva de desinversiones, derivadas de la crisis en la que se han visto envueltos multitud de países, que ha afectado a casi todos los sectores de la economía tanto de Europa como de Estados Unidos, y cuyo impacto ha derivado en unos procesos de reestructuración y configuración de las empresas.

Así la reestructuración de plantilla es "el proceso por el que cada empleado es asignado al puesto más apropiado, de manera que se aprovechen al máximo las competencias de los individuos de forma conjunta; pudiendo implicar o no cambios en la estructura organizativa y reducción de parte del personal" (Fernández Sánchez, 2005, citado por Fernández, 2006).

Los rápidos e intensos cambios sociales y tecnológicos del entorno están provocando que las estrategias de reestructuración organizativa o de reducción del

${ }^{3}$ Las Cajas de Ahorros y las cooperativas de crédito no se incluyen en nuestro estudio debido a la diferencia de tamaño de estas con los bancos. Hecho que es necesario tener presente ya que, aunque la utilización de ratios implica la eliminación automática del efecto de dimensión de una empresa, en numerosas ocasiones puede suceder que éste influya y por tanto el rango de valores sea distinto para las grandes empresas que para las PYMES. 
tamaño de la empresa se convierta en un fenómeno empresarial cada vez más importante y en un reto para la investigación en Dirección de Empresas.

Se puede considerar que la reestructuración de plantillas es una medida muy importante, si no la que más, de la política de personal de una empresa, debido a sus consecuencias tanto internas como externas, además, es uno de los mayores desafíos para los empresarios (Marr y García, 1997).

Es importante señalar, que el fenómeno de la reestructuración afecta a todos los tipos de organizaciones, tanto públicas como privadas, y a todas las categorías profesionales, es decir, se pueden ver afectados tanto el núcleo de operaciones de la organización como los ejecutivos de línea media o el personal de staff.

\subsubsection{Causas de la reestructuración empresarial}

Una reestructuración de plantilla supone una remodelación total de la estructura de la empresa provocada por cambios, tanto por las circunstancias económicas nacionales e internacionales como por las internas de las propias empresas (Arboledas et.al, 2002). Entre las primeras, provocadas por motivos ajenos al empresario, se encuentran la desaceleración de la economía del país; el desarrollo de las nuevas tecnologías de la información y de la comunicación; la corta vida de los productos; la globalización de los mercados; la lucha por la competencia y la existencia de un consumidor cada vez más exigente e infiel. Entre las segundas, provocadas por causas internas de la propia organización, destacan, entre otras, la mala gestión empresarial y una estructura organizativa inadecuada (sobredimensionamiento de la plantilla, falta de flexibilidad y de formación de los empleados, duplicación de tareas y puestos de trabajo). Por ello, debemos analizar, una a una, todas las causas que provocan las crisis empresariales, y en función de ellas plantear las alternativas oportunas, que no siempre tienen que pasar por iniciar un proceso de despido.

En un primer momento muchas investigaciones centraron su interés en explicar los antecedentes del downsizing en empresas que estaban atravesando una situación de crisis (Budros 1997, 2000, 2002; DeWitt, 1998; Dahl y Neshelm, 2010; Filatotochev et.al, 2000; Love y Nohria 2005; Ahmadjian y Robinson 2001) No obstante, progresivamente se han ido dando casos de empresas que gozan de un buen rendimiento financiero y que también han sido proclives a iniciar reducciones voluntarias de personal. Es decir que una situación de crisis puede desencadenar una reducción de la fuerza de trabajo (en cuyo caso se trataría de una reacción defensiva de la compañía); pero no siempre los procesos de reestructuración de plantilla se adoptan en estas empresas que han sufrido un declive y ésta no es la única causa de estos procesos, sino que también existen factores proactivos normalmente dirigidos a fortalecer la competitividad de la empresa (Sánchez y Suárez, 2005). 


\subsubsection{Principales métodos de reestructuración}

En base a la definición del término reestructuración (Fernández 2006) el fenómeno de la reestructuración "es el proceso por el que cada empleado es asignado al puesto más apropiado, de manera que se aprovechen al máximo las competencias de los individuos de forma conjunta; pudiendo implicar o no cambios en la estructura organizativa y reducción de parte del personal". Podemos diferenciar cuatro métodos de reestructuración de plantilla: los despidos laborales, las prejubilaciones, la movilidad funcional o geográfica y la recolocación de los empleados.

\section{A. Despido laboral}

El despido laboral es la acción a través de la cual un empresario o empleador da por finalizado unilateralmente el contrato laboral que mantenía con su trabajador o empleado. El Estatuto de los Trabajadores de España recoge tres motivos genéricos por los cuales un empresario puede despedir de forma objetiva, es decir con una causa justificada, a un empleado: por motivos económicos, de organización o técnicos. La diferencia entre un despido objetivo o justificado y uno improcedente se sitúa básicamente en la indemnización que perciben los trabajadores.

Los tipos de despido que existen son tres: el despido procedente, el despido improcedente y el despido nulo.

- Despido procedente: este tipo de despido está amparado en alguna causa que la ley y, en su caso, el contrato firmado, considera suficiente como para romper unilateralmente la relación. En este caso, el empleado no puede exigir indemnización puesto que el despido se hace dentro de los límites que marca la ley.

- Despido improcedente: se trata de un despido que no tiene causa, o en su defecto, dicha causa no está contemplada en la ley o en el contrato firmado entre las partes. El empleado normalmente puede exigir una indemnización por dicho despido, que dependerá de la legislación aplicable.

- Despido nulo: En ocasiones, ciertas legislaciones entienden que el despido por ciertas causas es nulo. En ese caso, el empresario está obligado a pagarle al empleado, o bien una indemnización, o bien a readmitirle de nuevo en la empresa con efectos retroactivos.

\section{B. Prejubilaciones}

Se consideran prejubilaciones a los procesos que afectan a las personas que en el momento de la interrupción de su actividad profesional remunerada no tienen ningún derecho, por edad, a cobrar ningún tipo de pensión proveniente de la Seguridad Social (Gómez y Martí 2003). En este tipo de procedimientos se establece un pacto entre el 
empleado y el empresario mediante el cual se indemniza al trabajador en unas condiciones favorables para que llegue a la edad de jubilarse sin problemas económicos.

Las prejubilaciones pueden concurrir de tres maneras diferentes.

- Indemnización al trabajador por parte de la empresa: el empleado puede recibirla en una única modalidad de pago en el momento del despido, o en varios pagos fraccionados que durarán hasta el momento en el que el trabajador alcance la edad legal de jubilación.

- Prestación por desempleo: en esta modalidad, el trabajador percibirá un sueldo con cargo al Instituto Nacional de Empleo (INEM) durante un periodo no superior a dos años. Durante este período el INEM se encargará de ingresar las cotizaciones a la Seguridad Social.

- Subsidio por desempleo: el trabajador cobrará un sueldo (75\% del salario mínimo interprofesional) hasta que alcance la edad legal de jubilación. Durante este periodo el INEM será el organismo encargado de ingresar las cotizaciones a la Seguridad Social.

\section{Movilidad FunCiOnAl y GEOGRÁficA.}

La movilidad tanto geográfica como funcional constituye uno de los instrumentos característicos de las técnicas de reestructuración de plantilla.

a) Movilidad funcional: se trata de un tipo de movilidad relativa a las funciones o al lugar de prestación de servicios. Se pueden distinguir dos tipos de movilidad dentro de esta modalidad:

- Movilidad dentro del grupo profesional o entre categorías equivalentes.

- Movilidad fuera del grupo o entre categorías no equivalentes.

b) Movilidad geográfica: este tipo de movilidad se justifica en base a cambios geográficos ${ }^{4}$ fundados en razones económicas, técnicas organizativas y de producción. Aunque puede ser de muy diversos tipos, destacan principalmente dos: traslados y desplazamientos.

\section{ReCOLOCACiÓN DE EMPLEAdOS}

La recolocación de empleados ${ }^{5}$ se define como el servicio que una empresa aporta a sus trabajadores que han sido despedidos a través del cual se les ofrece la posibilidad de recibir asesoramiento, tanto por consultores externos como por sus

4 Según el Estatuto de los Trabajadores (en adelante ET) son cambios geográficos cuya duración sobrepasa los 12 meses dentro de un período de 3 años. Pueden ser individuales o colectivos.

${ }^{5}$ Término conocido en la literatura anglosajona como outplacement. 
propios medios, formación y los medios necesarios para lograr una transición en su carrera profesional, de manera que puedan conseguir un nuevo trabajo en otra empresa lo más adecuado posible a sus actitudes y aptitudes en el menor plazo posible, de manera que se consiguen reducir los perjuicios económicos y psicológicos que suponen todas las situaciones de despido (Sastre, 1995).

En España, la recolocación se enmarca en las medidas inmersas en los denominados Planes de Acompañamiento Social (PAS) previstos en el Real Decreto 43/1996 sobre Reglamento de los Procedimientos de Regulación de Empleo y de Actuación Administrativa en Materia de Traslados Colectivos, que establece el procedimiento de regulación de empleo fundado en causas económicas, técnicas, organizativas o de producción (Hortal, 2006).

En la tabla 1 se muestran, a modo de resumen, los distintos métodos de restructuración empleados por las empresas así como algunos aspectos principales de los mismos.

\section{Tabla 1. Métodos de reestructuración}

\begin{tabular}{|c|c|c|c|c|}
\hline & Despidos & Prejubilaciones & Movilidad & $\begin{array}{l}\text { Recolocación de } \\
\text { empleados }\end{array}$ \\
\hline$\stackrel{\infty}{\stackrel{0}{0}}$ & $\begin{array}{l}\text { - Procedente } \\
\text { - Improcedente } \\
\text { - Nulo }\end{array}$ & $\begin{array}{l}\text { - Indemnización } \\
\text { por parte de la } \\
\text { empresa } \\
\text { - Prestación por } \\
\text { desempleo } \\
\text { - Subsidio por } \\
\text { desempleo }\end{array}$ & $\begin{array}{l}\text { - Funcional } \\
\text { - Geográfica }\end{array}$ & \\
\hline 节 & Empleados & Empleados & Actividades & $\begin{array}{l}\text { Empleados y } \\
\text { actividades }\end{array}$ \\
\hline 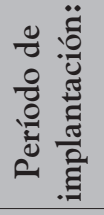 & Rápido & Moderado & Moderado & Moderado/largo \\
\hline 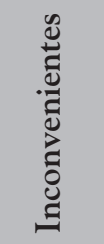 & \multicolumn{2}{|c|}{$\begin{array}{l}\text { No se obtienen } \\
\text { resultados inmediatos }\end{array}$} & $\begin{array}{c}\text { No se ahorran } \\
\text { costes a corto } \\
\text { plazo }\end{array}$ & $\begin{array}{c}\text { Falta de } \\
\text { adaptación e } \\
\text { incremento de } \\
\text { costes }\end{array}$ \\
\hline
\end{tabular}

Fuente: elaboración propia a partir de Sastre (1995), Gómez y Martí (2003), Fernández (2005) y Hortal (2006). 
Dentro de la literatura española encontramos algunos estudios más recientes que tratan el problema del redimensionamiento empresarial, como es el caso de la publicación de la fundación para el desarrollo de la función de Recursos humanos, en el que, según los autores, estos procesos pueden suponer un coste o una inversión para la empresa en función de cómo se realice la gestión del mismo. En nuestro país, el cumplimiento de estos planes es relativamente novedoso, causa de ello es la inexperiencia por parte de las empresas a la hora de recurrir a estos planes.

Por otro lado, en la literatura anglosajona existen diferentes corrientes de pensamiento respecto al tema de la reestructuración, la gran mayoría de autores, que lo han estudiado, coinciden en la utilización de este término para describir cualquier modificación que se produce en la estrategia de gestión de la empresa.

Existen pues, dos corrientes de autores que explican el proceso de reestructuración de plantillas desde dos puntos de vista diferenciados (Fernandez, 2006): los autores anglosajones identifican con este término el proceso general que hace que una empresa reduzca su tamaño, con todas las implicaciones que de ello se derivan, fundamentalmente en el número de trabajadores (Cameron, 1994; McKinley et.al, 1998; Budros, 1999, entre otros).En este sentido, se propone un modelo con diferentes estrategias de downsizing en función de la extensión de su aplicación (Cameron, 1994). Estas estrategias se configuran de la siguiente manera (ver tabla 2):

- Reducción de la fuerza de trabajo: Cameron propone una reducción de los empleados de la organización de una manera rápida, de manera que los resultados puedan ser visibles a corto plazo. Este tipo de estrategia se basa en la realización de acciones tales como, prejubilaciones, traslados y despidos, entre otras. Estas acciones normalmente suelen implantarse en la empresa desde los altos cargos a los más bajos en la jerarquía organizativa. Con ello, se busca atraer la atención de los empleados, de manera que sean consciente de la difícil situación en la que se encuentra inmersa la empresa. Así, se consigue un ahorro de costes diario. Sin embargo, esta estrategia no está exenta de inconvenientes, ya que puede suponer la pérdida de personal valioso para la organización.

- Rediseño de los procesos de trabajo: el interés principal de esta estrategia emerge en la búsqueda de una reducción de las actividades, niveles y unidades organizativas, para lograr una reducción del número de trabajadores. Este tipo de acciones se implantan a medio plazo puesto que es necesario realizar importantes modificaciones en la empresa.

La principal forma de llevar a cabo esta estrategia es mediante la eliminación de staff, la combinación de unidades organizativas o el rediseño de tareas. Así, se consigue un aumento de la eficiencia debido a la simplifica- 


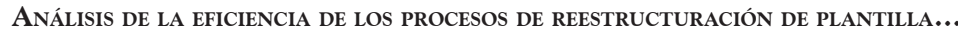

Tabla 2. Estrategias de downsizing en función de su aplicación

\begin{tabular}{|c|c|c|c|}
\hline & $\begin{array}{l}\text { Reducción de la fuerza } \\
\text { de trabajo }\end{array}$ & $\begin{array}{l}\text { Rediseño de los } \\
\text { procesos de trabajo }\end{array}$ & Modificación sistémica \\
\hline 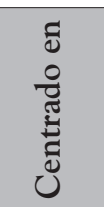 & Personal & $\begin{array}{c}\text { Trabajo, niveles y } \\
\text { unidades organizativas }\end{array}$ & Sistémico \\
\hline $\begin{array}{l}\tilde{G} \\
\text { 节 }\end{array}$ & Empleados & Actividades & Status quo \\
\hline 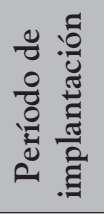 & Rápido & Moderado & Largo \\
\hline 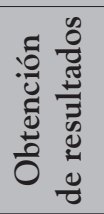 & Corto plazo & Medio plazo & Largo plazo \\
\hline 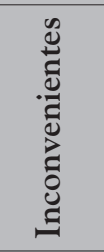 & $\begin{array}{l}\text { Falta de adaptación a } \\
\text { largo plazo }\end{array}$ & $\begin{array}{l}\text { No se obtienen } \\
\text { resultados inmediatos }\end{array}$ & $\begin{array}{c}\text { No se ahorran costes a } \\
\text { corto plazo }\end{array}$ \\
\hline 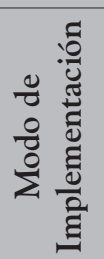 & $\begin{array}{l}\text { Despidos, } \\
\text { prejubilaciones, } \\
\text { jubilaciones }\end{array}$ & $\begin{array}{l}\text { Rediseño de tareas, } \\
\text { eliminación de staff. }\end{array}$ & $\begin{array}{l}\text { Implica a toda la } \\
\text { organización y a toda la } \\
\text { cadena de valor. }\end{array}$ \\
\hline
\end{tabular}

Fuente: adaptado de Cameron, 1994

ción de la estructura de la organización. Al igual que ocurría con la implantación de la estrategia anterior, entre los inconvenientes que se pueden dar, encontramos la aparición de problemas de desmotivación y sobrecarga de trabajo en los empleados. Para ello, es necesario realizar un exhaustivo estudio de la organización y analizar cómo se van a llevar a cabo esas acciones. 
- Modificación sistémica: el autor propone una tercera estrategia basada en la modificación de la cultura empresarial, así, propone acciones como una reducción de los costes asociados a proveedores y a clientes o la realización de una integración del sistema de información. La mayor ventaja de este tipo de estrategias es que facilitan el ahorro de costes en toda la cadena de valor empresarial. Su principal desventaja es que este tipo de acciones es necesario que se implanten en la organización a largo plazo, puesto que un cambio de cultura empresarial requiere un importante análisis por parte de la organización.

En base a esta clasificación, el término downsizing se sitúa dentro de las modificaciones sistémicas, al ser una alternativa estratégica que comprende distintas combinaciones de disminuciones en los sistemas físicos, humanos y organizativos de una empresa para ajustarla a las condiciones competitivas de una unidad de negocio (DeWitt, 1998). Este conjunto de acciones, diseñadas e implantadas por la dirección, tienen como objetivo la mejora de eficiencia, productividad y/o competitividad de una organización, influyendo de forma directa en el tamaño, costes y procesos de trabajo de cada unidad organizativa.

Las principales características del downsizing como estrategia de reducción selectiva de un conjunto de recursos de la organización son (Cameron et.al, 1993):

- Personal: Implica una reducción del tamaño de la unidad organizativa, lo que conlleva una disminución de la plantilla (mediante prejubilaciones y despidos, entre otros).

- Momento: El downsizing puede implantarse de forma proactiva o reactiva, en función de si se busca un mecanismo de ataque o de defensa, respectivamente.

- Procesos de trabajo: esta estrategia busca la modificación de los sistemas fundamentales de la organización. En este sentido, es necesario llevar a cabo un proceso de rediseño en la organización, como consecuencia de la alteración de las tareas o actividades, de las unidades organizativas de los y niveles jerárquicos de la organización.

Sin embargo, existe otra corriente de pensamiento para quienes la reducción de plantilla es sólo un aspecto de las muchas técnicas que lleva consigo el proceso de downsizing. (Bowman y Sing, 1993; Band y Tustin, 1995; Whetten, y Godfrey, 1998 y Pichault y Rorive, 2003).

Otra clasificación de los tipos de reestructuración que pueden llevarse a cabo en una organización (Bowman y Singh, 1993):

- Reestructuración financiera: implica cambios en la estructura del capital de la empresa principalmente provocada por los procesos de adquisiciones y fusiones. 
- Reestructuración corporativa: en este tipo de reestructuración los principales cambios se ven reflejados en la composición de la cartera de negocios, bien sea abandonando carteras de productos y mercados o incorporando nuevas gamas de productos y nuevos mercados en la estrategia.

- Reestructuración organizativa: implica cambios en la estructura organizativa de la empresa que se llevan a cabo modificando los mecanismos tradicionales del diseño organizativo de la empresa.

En esta misma línea, se diferencian otros aspectos dentro de este campo, indicando que una reestructuración no siempre implica despidos, sino que puede significar simplemente una reasignación de personas a puestos con el fin de racionalizar los procesos y actividades de la empresa con el ánimo de mejorar su situación económica (Freeman, 1994).

\subsection{Problemas de los métodos de reestructuración de plantilla}

La reestructuración de la plantilla se puede explicar, si tenemos en cuenta las dos corrientes de pensamiento, como un proceso de reorganización de los recursos de la empresa con el fin de asegurar su supervivencia. Así pues, cuando se lleva a cabo este tipo de procesos, pueden plantearse dos problemas diferentes (Fernández, 2006):

- Si se trata de la reducción de una parte de la plantilla, esto podría suponer para la empresa la pérdida de ciertos recursos y capacidades necesarios para su supervivencia y éxito (Casillas y Barroso, 1998). Además, en este caso se corre también el riesgo de que los trabajadores que se quedan vean su puesto en la empresa amenazado y sientan inseguridad, falta de confianza y desmotivación, lo cual repercutirá en un menor rendimiento y productividad y por lo tanto en una peor calidad del producto o servicio.

- Por otro lado, si lo que se produce es una reorganización de la empresa, se corre el riesgo de infrautilizar los recursos y capacidades de los que se dispone, si no se hace adecuadamente.

Estos dos aspectos obligan a hacer una breve referencia a la gestión del conocimiento, especificando algún aspecto relativo a la reestructuración de plantillas, dado que no todos los empleados poseen las mismas habilidades y por tanto no poseen, tampoco, el mismo conocimiento. Dentro del ámbito de la gestión del conocimiento, se distinguen dos tipos, conocimiento genérico y específico y el conocimiento tácito y explícito. El conocimiento genérico es el más fácil de obtener y de copiar, por lo tanto es el que menos interesa a la empresa puesto que éste no 
va a hacer que se distinga de sus competidores. Por el contrario, el específico no está disponible en el mercado sino que se desarrolla dentro de la empresa (Bueno, 1998). Es este conocimiento el que la empresa debe esforzarse o cultivar y desarrollar, de manera que no se pierda si un empleado se ve afectado por el proceso de reestructuración. Por otro lado, el conocimiento explícito es aquel que se encuentra formalizado en manuales y fórmulas, entre otros, y es fácil de transmitir.

Por último, es importante conocer el conocimiento tácito ya que éste es más difícil de formalizar puesto que se encuentra en las personas y es una filosofía o manera de hacer las cosas muy específica; por lo tanto, su transmisión es complicada y de ahí su importancia a la hora de llevar a cabo los procesos de reestructuración en la empresa (Nonaka y Takeuchi, 2007).

Además, es importante tener en cuenta que si una decisión de esta naturaleza no se maneja de forma adecuada, afectará negativamente al proyecto empresarial de futuro, desde el punto de vista de credibilidad interna y externa, pudiendo constituir un elemento que utilice la competencia para ganar cuotas de mercado aprovechándose de la situación de conflicto de la empresa, a la vez que pueden redundar en una pérdida de imagen corporativa difícil de recuperar una vez que se supere esta situación negativa (Martín, 2007).

Las principales ventajas e inconvenientes de los distintos métodos de reestructuración de plantilla llevados a cabo en las organizaciones quedan detalladas en la tabla 3.

\subsection{Eficiencia en los procesos de reestructuración}

Numerosos autores atribuyen a las palabras eficiencia y productividad, un significado idéntico: "productividad es la eficiencia con la cual se han utilizado los recursos para elaborar productos y servicios a niveles específicos de calidad y de manera oportuna" así (Dulworth, 1991). En la realidad empresarial la definición más común de productividad se basa en la relación que existe entre los productos o resultados y los recursos que se consumieron para su obtención (Koontz y Weilhrich, 2008).

Otros autores definen la eficiencia como "la capacidad de obtener los mejores resultados con la mínima inversión. Se define como hacer bien las cosas" (Robbins y Coulter, 2005). "La eficiencia se refiere a la relación entre esfuerzos y resultados" (Diez et. al, 2002). "La eficiencia es un término orientado a los medios utilizados para alcanzar los objetivos (Iborra et. al,2007). La eficiencia consiste en "alcanzar los fines con el mínimo de recursos" (Koontz et. al, 2008).

En el ámbito de las ciencias sociales suele definirse la eficiencia como la relación existente entre los resultados obtenidos y los medios utilizados para conseguirlos. Así pues, el concepto de productividad (o eficiencia) no es una medida del volumen de producción sino de la capacidad que tiene un sistema para inte- 


\section{Tabla 3. Ventajas e inconvenientes de los métodos de reestructuración de plantilla}

\begin{tabular}{|c|c|c|}
\hline & VENTAJAS & INCONVENIENTES \\
\hline 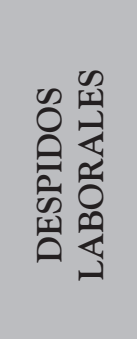 & $\begin{array}{l}\text { - Disminución de costes para el } \\
\text { empresario. } \\
\text { - Libertad del empresario. } \\
\text { - Aumentos en la productividad de } \\
\text { los empleados. }\end{array}$ & $\begin{array}{l}\text { - Rigidez legislativa en algunas } \\
\text { ocasiones. } \\
\text { - Puede resultar traumático para los } \\
\text { empleados y propiciar inseguridad, } \\
\text { desconfianza y desmotivación. } \\
\text { - En ocasiones puede ocasionar la } \\
\text { pérdida de recursos y capacidades } \\
\text { necesarios para la empresa. }\end{array}$ \\
\hline 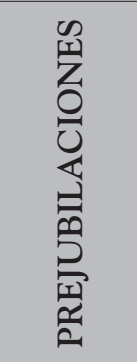 & $\begin{array}{l}\text { - Compensación del salario. } \\
\text { - Rejuvenecimiento de la plantilla. } \\
\text { - Buena acogida entre algunos } \\
\text { empleados. } \\
\text { No traumática entre los } \\
\text { empleados. }\end{array}$ & $\begin{array}{l}\text { - Elevado coste para la empresa. } \\
\text { - En ocasiones, dependiendo del } \\
\text { tipo de empleado, puede resultar } \\
\text { traumática. } \\
\text { - Rigidez legislativa. } \\
\text { Salida precoz de la vida activa } \\
\text { laboral. }\end{array}$ \\
\hline 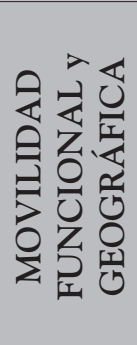 & $\begin{array}{l}\text { - Mejora el nivel de preparación de } \\
\text { los empleados. } \\
\text { - Libertad del empresario y } \\
\text { - flexibilidad. } \\
\text { - Aumenta el rendimiento de los } \\
\text { empleados. } \\
\text { - Moderniza la estructura de la } \\
\text { empresa. }\end{array}$ & $\begin{array}{l}\text { - Elevado gasto en formación. } \\
\text { - En ocasiones, puede suponer } \\
\text { una desmotivación por parte del } \\
\text { empleado. } \\
\text { - Reticencia en su aplicación }\end{array}$ \\
\hline 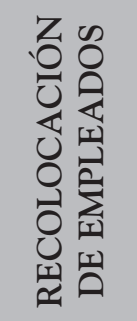 & $\begin{array}{l}\text { - Mejora la imagen de la empresa. } \\
\text { - Mejora el clima laboral } \\
\text { - Evita la desmotivación. } \\
\text { - Suaviza las consecuencias del } \\
\text { despido. } \\
\text { - Disminuye el gasto de la } \\
\text { administración pública. }\end{array}$ & $\begin{array}{l}\text { - Mayor coste para la empresa. } \\
\text { - En ocasiones puede parecer } \\
\text { discriminatorio. }\end{array}$ \\
\hline
\end{tabular}

Fuente: adaptado de Arboledas, Hortal y de Enterría Pérez, 2002.

grar y combinar los recursos humanos, físicos y financieros que intervienen en la elaboración de un producto, con el propósito de utilizarlos económicamente. El 
aumento de la productividad ayuda a conservar los recursos escasos y costosos en las empresas.

Cuando se conduce una investigación sobre temas de máxima actualidad, tal y como probablemente ha estado ocurriendo con el downsizing, a menudo nos encontramos con que no existe una definición comúnmente aceptada de su concepto, ámbito de aplicación y consecuencias, lo que dificulta el tratamiento de la información existente. A pesar de que las prácticas de downsizing se han generalizado a nivel mundial en las grandes empresas existen todavía muchos interrogantes sobre este tema puesto que no se ha podido comprobar efectivamente que los efectos de estas técnicas sean los perseguidos inicialmente. Una de las cuestiones más debatidas dentro de la literatura con estudios empíricos sobre el downsizing es la confirmación o, en su defecto, el rechazo de la hipótesis sobre la eficiencia económica de estas técnicas, esto es, si las empresas que hacen reestructuración de plantilla consiguen mejorar sus resultados económicos y organizativos. A este respecto, la evidencia disponible está lejos de ser concluyente ya que existen trabajos que lo asocian, por un lado, a mejores resultados y, por otro, a efectos negativos.

Siguiendo las investigaciones realizadas por diversos autores (Huselid, 1995; De Meuse et. al, 2004; Love y Nohria, 2005; Yu y Park, 2006; Love y Kraatz, 2009; Sheaffer et.al, 2009; Odette et.al, 2009; Muñoz y Sanchez, 2010 y 2011), entre otras, los indicadores o variables que serán utilizados en esta investigación para determinar si una empresa que ha llevado a cabo un proceso de reestructuración de plantilla ha logrado o no mejorar su eficiencia son: la rentabilidad financiera $(\mathrm{ROE})$, la rentabilidad económica (ROA), la ratio de eficiencia, la productividad por empleado o beneficio medio conseguido por trabajador y el coste medio por trabajador

\section{METODOLOGÍA}

\subsection{Diseño de la investigación}

El criterio seguido para la elección del universo de estudio ha sido el número de empleado, ya que si se querían estudiar los efectos sobre la reestructuración de personal, esa debía ser la base de la investigación. Para ello, siguiendo las investigaciones anteriores, nos centramos en empresas de tamaño grande, es decir, aquellas que contaban con una cifra de empleados igual o superior a $250^{6}$ (Budros 1997;Suárez,1999; Suárez y Vicente, 2000).

En cuanto al sector objeto de estudio, nos centramos en el sector financiero español y más concretamente en el caso particular de los bancos que cotizaban

\footnotetext{
${ }^{6}$ Clasificación de la empresa según la UE.
} 
en el IBEX 35 en el momento de la investigación. Los motivos principales por los que se escogieron estas entidades para realizar el estudio radicaron en la accesibilidad y la veracidad de la información de estas entidades, puesto que sus cuentas anuales eran públicas y además, estaban auditadas y supervisadas por el Banco de España.

Así, en función de estas variables: empresas grandes, del sistema financiero español y que coticen en el IBEX 35, se obtuvo una muestra de cinco empresas, en concreto cinco bancos: BBVA, Bankinter, Banco Popular, Banco Sabadell y Banco Santander. Así, con esta muestra, el objetivo principal de la investigación radicaba en analizar la eficiencia de los procesos de reestructuración de plantilla de estas empresas, es decir, cómo afectaban las variaciones de plantilla de estas cinco entidades a sus resultados económicos.

El periodo escogido para realizar el análisis ha abarcado desde los años 2003 hasta 2012, ambos incluidos. De esta manera, al analizar dos bloques diferenciados en cuanto a la situación económica del país (2003-2007), periodo en el que nos encontrábamos en años de bonanza económica y el segundo período (20082012) período en el que estalló la crisis económica y financiera, se podrían analizar los efectos que dicha crisis había tenido sobre estas organizaciones al tener que realizar las técnicas de reestructuración de plantilla para intentar mejorar su eficiencia.

Se ha realizado un análisis cuantitativo con el que se ha procedido al tratamiento de los datos financieros obtenidos por las empresas que constituían el objeto de estudio en esta investigación. Los datos se introdujeron para su tratamiento estadístico en el programa Stata. Se trataba de un programa estadístico y econométrico muy potente para analizar, manejar y representar gráficamente los datos analizados. El motivo de la elección de este programa frente a otros programas fue la rapidez en el proceso y en el tratamiento de los datos. Una vez se obtuvieron los resultados, proporcionados por el programa, se realizó un análisis comparativo de los datos obtenidos y de los indicadores financieros de cada una de las empresas (beneficio y principales ratios económico-financieros), de manera que pudiera analizarse en conjunto la eficiencia de los procesos de reestructuración de plantilla.

La naturaleza de este estudio es dinámica, por lo que resultaba más apropiada la utilización de datos de panel, ya que estos contienen observaciones de determinadas variables para todas las empresas de la muestra en cada uno de los años comprendidos en el período a analizar. Además, la principal ventaja de este tipo de datos es que permiten contrastar el modelo controlando la existencia de heterogeneidad inobservable, es decir, que permite ver el efecto individual de todas las características de la empresa que no se han podido medir (Magán y Céspedes, 2007).

El modelo econométrico utilizado para la realización del análisis empírico fue un modelo de regresión lineal con datos de panel (Huselid, 1995; Bruton et.al 
1996; Budros, 1997; Cascio, 1997; O’Shaughnessy y Flanagan, 1998; Suárez,1999; Suárez y Vicente, 2000; Yoo y Mody, 2000; Espahbodi et.al, 2000; McElroy et.al, 2001; Baumol et.al, 2003; De Meuse et.al, 2004; Love y Nohria, 2005; Perry y Shivdasani, 2005; Magán y Céspedes, 2006; Yu y Park, 2006; Coucke et.al, 2007, Guthrie y Datta, 2008; Mellahi y Wilkinson, 2010; Sheaffer et.al 2009; Love y Kraatz, 2009; Sheaffer et.al, 2009; Odette et.al, 2009; Muñoz y Sanchez, 2010 y 2011), puesto que este tipo de modelo era el más apropiado para medir la relación causal entre una muestra de agentes económicos (como son las empresas en el caso que nos concernía) para un determinada periodo de tiempo.

Así, esta técnica nos permitió realizar un análisis más dinámico al incorporar la dimensión temporal de los datos, lo cual consentía enriquecer el estudio, especialmente en periodos de grandes cambios, como era el caso.

En el modelo que se planteó la variable dependiente (Y) era la eficiencia medida a través de las variables más utilizadas comúnmente para medirla, que tal y como se ha comentado anteriormente se midió con la rentabilidad económica, la rentabilidad financiera, el EBITDA, el ratio de eficiencia, la productividad por trabajador y el coste medio por empleado; y las variables explicativas $(\mathrm{X})$ fueron tanto la variación de la plantilla como dos variables de control que se han añadido siguiendo a diversos autores que fueron el tamaño de la empresa (Budros, 1997; Suárez, 1999; Suárez y Vicente, 2000; McElroy et.al, 2001; De Meuse, et.al, 2004; Love y Nohra, 2005; Magán y Céspedes, 2006; Love y Kraatz, 2009; Mellahi y Wilkinson, 2009; Sheaffer et.al, 2009; Muñoz y Sanchez, 2011; Yu y Park, 2006) medido con el volumen de activos, con la plantilla y con el número de sucursales y el valor de mercado de la empresa.

Así nuestra ecuación quedo de la siguiente manera:

$$
\text { EFICIENCIA }_{\mathrm{it}}=\alpha_{\mathrm{i}}+\beta \text { dplantilla }_{\mathrm{it}}+\mathrm{Y}_{1} \text { tamaño }_{\mathrm{it}}+\mathrm{Y}_{2} \text { valormercado }_{\mathrm{it}} \epsilon_{\mathrm{it}}
$$

Esta metodología admitía elegir entre dos métodos diferentes: el modelo de efectos fijos y el modelo de efectos aleatorios. El modelo de efectos fijos garantiza estimaciones consistentes aunque exista correlación entre los represores y el error específico a la unidad o al grupo, mientras que el modelo de efectos aleatorios es más eficiente siempre que las variables aleatorias no estén correlacionadas con los regresores (Magán y Céspedes, 2007).

Para saber cuál de los dos era el modelo más útil para nuestro análisis se realizó el test de Hausman con objeto de escoger entre el modelo de efectos fijos y el de efectos aleatorios; básicamente este test permite conocer si las variables endógenas incluidas en una ecuación como explicatorias están o no correlacionadas con el termino de perturbación aleatoria. El estadístico de prueba desarrollado por Hausman presenta una distribución asintótica $X^{2}$. En caso de que dicha correlación resulte ser diferente de cero, se asume la presencia de simultaneidad entre 
las ecuaciones del sistema. Así, en función de los resultados obtenidos diremos lo siguiente:

- H=0 No hay Simultaneidad (la correlación entre la variable regresora endógena y el término de error es cero asintóticamente).

- Hキ0 Hay Simultaneidad (la correlación entre la variable regresora endógena y el término de error es diferente de cero).

Si se rechaza la hipótesis nula, la conclusión es que el modelo de efectos aleatorios no resulta adecuado y que convendría más emplear el modelo de efectos fijos. Para comprobarlo, se realizó el test con cada una de las 6 variables utilizadas para medir la eficiencia empresarial. Los resultados obtenidos en este test nos indicaron que la mejor opción era la estimación por efectos aleatorios, pues la significación de la variable plantilla era superior cuando se realizaba con este tipo de estimación, lo que significaba que aceptábamos la hipótesis nula y que, por tanto, el modelo que se debía emplear era el de efectos aleatorios.

\section{RESULTADOS}

Se han realizado distintas estimaciones con varios modelos diferenciados. Para ello se han analizado 3 modelos utilizando las variables de control tamaño de la empresa con cada una de las formas en las que se puede medir esta variable (plantilla, volumen de activos y sucursales) y valor de mercado para cada una de las medidas de eficiencia utilizadas. La necesidad de utilizar variables de control radica en que es un tipo de variable independiente que no se manipula sino que se mantiene constante con el objeto de neutralizar sus efectos sobre la variable dependiente, puesto que en una investigación no es posible estudiar simultáneamente todas las variables independientes. Por lo tanto como tenemos 6 medidas de la eficiencia y 3 modelos para cada una de las variables que miden el tamaño, se han obtenido 18 modelos distintos. Además, tras realizar una matriz de correlaciones de todas las variables que miden el tamaño de la empresa, se ha observado que las correlaciones entre las tres variables son muy elevadas, tal y como se puede ver a continuación en la tabla 4 , hecho que justifica crear una variable única, Firmsize, que engloba a estas tres variables.

De manera que se han realizado seis modelos más para cada ratio medidor de la eficiencia con esta variable de control. Con lo cual, finalmente se han estimado 24 modelos diferentes.

Posteriormente, tras analizar los resultados de cada uno de los parámetros arrojados por el programa, se observó que solo se encontraba significación en tres de las seis variables analizadas: EBITDA, ratio de eficiencia y coste medio por 
Tabla 4. Correlaciones en las tres variables de tamaño

\begin{tabular}{|c|c|c|c|c|}
\cline { 2 - 5 } \multicolumn{1}{c|}{} & Plantilla & Activos & Sucursales & dplantilla \\
\hline Plantilla & 1,0000 & & & \\
\hline Activos & 0,7795 & 1,0000 & & \\
\hline Sucursales & 0,9617 & 0,8242 & 1,0000 & \\
\hline dplantilla & $-0,2312$ & $-0,2312$ & $-0,1827$ & 1,0000 \\
\hline
\end{tabular}

Fuente: elaboración propia a partir del programa Stata.

empleado. El motivo de descartar las otras tres variables (ROA, ROE y Productividad de los trabajadores) venía dado porque al realizar la estimación individual de cada uno de los modelos, en los resultados obtenidos, el resultado del p-value que se obtuvo sobre la variable dependiente era superior al $5 \%$, lo que indicaba que no había significatividad entre las variables, es decir, no se encontró ninguna relación entre la reducción de plantilla y las rentabilidades económica y financiera y con la productividad o el beneficio por trabajador.

Una vez que se escogieron las tres variables independientes en las que se encontraba relación con la variable dependiente, se realizó el test de Hausman con cada una de las tres variables para determinar qué modelo (modelo de efectos fijos o modelo de efectos aleatorios) era el más indicado para utilizar en esta investigación. Así una vez que se analizaron los datos mediante el análisis de regresión logística con los dos modelos se optó por el modelo de efectos aleatorios, ya que este permitía incrementar, sensiblemente, el número de observaciones utilizadas en la estimación.

Posteriormente, se estimó el modelo final, en el que se obtuvieron unos resultados al contrastar el modelo propuesto, tal y como podemos apreciar en la tabla 5, la variable dependiente plantilla resultaba significativa a un coeficiente del 10\% respectivamente, sobre la variable independiente EBITDA. En cuanto a la variable ratio de eficiencia no se obtuvo significación con la variable dependiente. Con respecto a la significatividad que se observó en la variable plantilla sobre la independiente medida con el coste medio, se puede observar que fue un nivel del 5\%.

En cuanto a las variables de control, firmsize y valor de mercado, la primera de ellas resultó ser significativa a un nivel del 1\% sobre las variables independientes 
EBITDA y coste medio por trabajador, mientras que la otra variable de control, valor de mercado, únicamente resultó significativa a un nivel del 10\% sobre la variable independiente ratio de eficiencia.

En cuanto al coeficiente $\mathrm{R}^{2}$, que es el encargado de medir la proporción de variación en la variable dependiente (plantilla) explicada por la variación en las variables independientes (EBITDA, ratio de eficiencia y coste medio por trabajador), los resultados que se obtuvieron es que la $\mathrm{R}^{2}$ explicada para cada una de las tres variables independientes era del 85\%, 19\% y 61\%, respectivamente, lo que advertía que en la variable EBITDA podría aceptarse que existía una buena correlación lineal, es decir que la variable EBITDA explicaba un 85\% de la variación de plantilla; mientras que en la segunda variable, ratio de eficiencia, el nivel de correlación que se obtuvo era bastante bajo e incluso nulo. En cuanto a la variable coste medio por trabajador podría decirse que esta variable explicaba que se produzca reducción de personal, en las empresas objeto del estudio, en un nivel de confianza del $61 \%$.

En la tabla 5 se exponen en primer lugar, en la primera columna, la variable dependiente y posteriormente aparecen las variables de control, así como otra información del estudio estadístico; mientras que en la primera fila del modelo final se puede observar las variables independientes que se han analizado como explicativas del downsizing. Las columnas encabezadas por las tres variables independientes proporcionan los coeficientes de significación para cada una de las variables que se han obtenido con el modelo de regresión lineal.

\section{Tabla 5. Resultados del modelo}

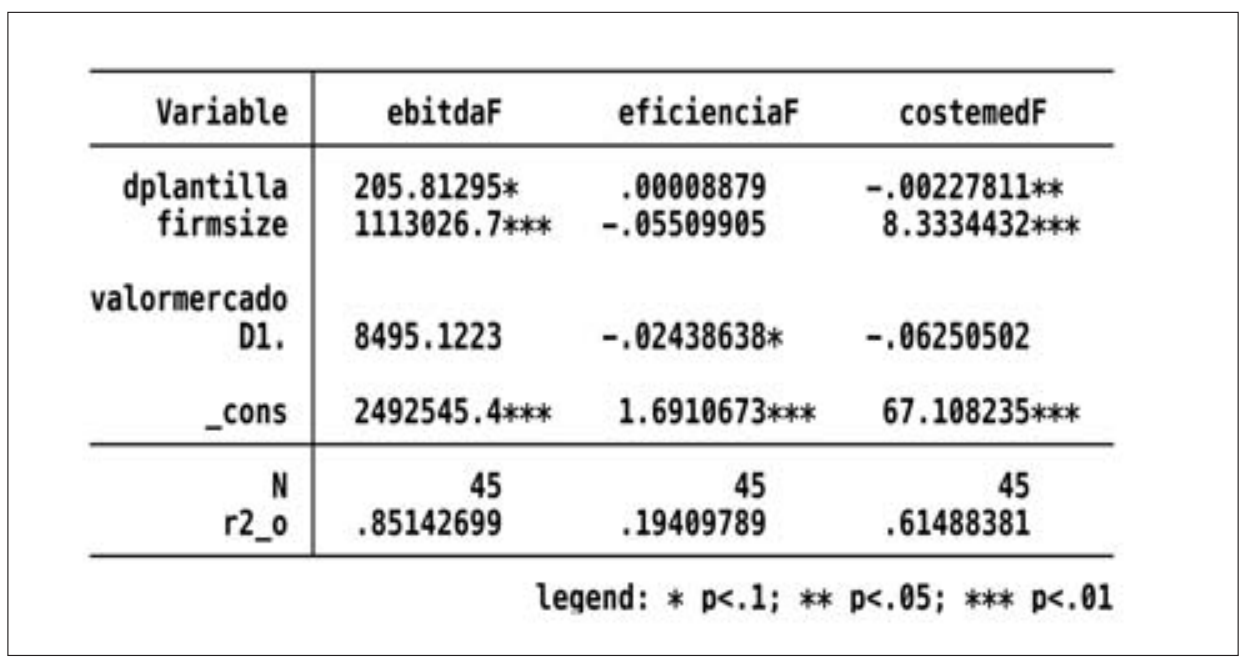

Fuente: elaboración propia a partir del programa Stata. 
A tenor de los resultados obtenido podemos decir que las compañías que implementan las técnicas de downsizing, no logran mejores resultados, ya que, tal y como muestra nuestro análisis, las empresas que llevan a cabo estrategias de reducción de plantilla no consiguen mejorar sus resultados medidos a través de los distintos indicadores utilizados para medir la eficiencia en tres de sus variables utilizadas, EBITDA, ratio de eficiencia y coste medio por trabajador. De manera que queda latente que hay evidencias que confirman una relación positiva entre el aumento de plantilla y el aumento de la eficiencia empresarial.

\section{Figura 1. Resultados de la investigación}

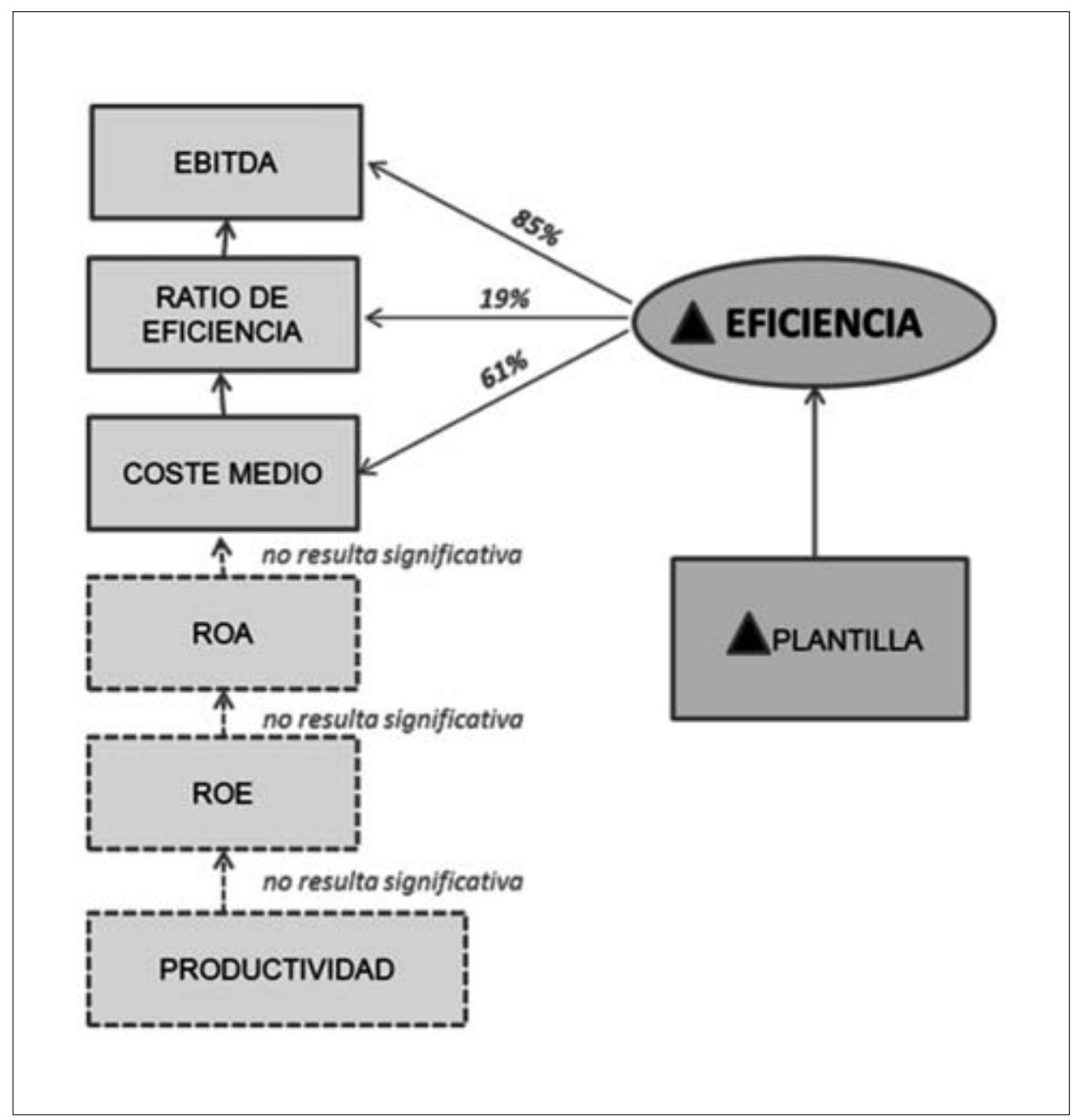

Fuente: elaboración propia. 


\section{CONCLUSIONES}

El presente apartado presenta las principales conclusiones e implicaciones sobre los resultados obtenidos en este estudio.

1. Reforzando las principales conclusiones obtenidas en trabajos previos sobre el downsizing en el ámbito español (Requejo, 1996; Suárez, 1999; Sanchez y Suarez, 2004; Claver y Fernández, 2006; Fernández, 2006; Odette et.al, 2007; Suárez y Vicente, 2009, Muñoz y Sánchez, 2010 y 2011), puede deducirse que la reducción de plantillas es una estrategia de gran magnitud utilizada frecuentemente entre las grandes empresas españolas. Además, queda de manifiesto en los estudios realizados con anterioridad, que el ciclo económico afecta de forma decisiva a la implementación de estas técnicas, puesto que tal y como se ha podido observar en los resultados obtenidos por estas cinco entidades, los años en los que la crisis económica (a partir del 2008) es más acusada, son también aquellos en los que existe una disminución más acusada de su número de empleados.

2. De ninguno de los resultados obtenidos en los modelos expuestos que examinan la relación entre downsizing y el aumento de la eficiencia de las empresas podemos concluir que el impacto sea positivo, ya que no se aprecian diferencias significativas entre la reducción de personal y la eficiencia empresarial. De hecho, a tenor de los resultados obtenidos, en los que la variación de plantilla resultaba significativa en tres de las variables dependientes a unas tasas de entre el 5 y el 10\%, queda de manifiesto que la eficiencia aumenta cuando la plantilla de las empresas se incrementa, pues el resultado que se obtuvo en el modelo fue positivo. Por lo que debemos rechazar la afirmación de que las grandes empresas que implementan estas técnicas consigan mejores resultados, ya que tal y como muestra nuestro análisis las empresas que llevan a cabo estrategias de reducción de plantilla no consiguen mejorar sus resultados económicos.

3. En este estudio hay evidencias de que existe una relación positiva entre tamaño y downsizing coincidiendo así con las conclusiones aportadas por otros autores (Budros, 1997; Suárez, 1999; Suárez y Vicente, 2000), las empresas con mayor tamaño experimentan una "necesidad de cambio" que obedece a redundancias e ineficiencias.

4. Los resultados obtenidos deben enmarcarse dentro de las limitaciones que presenta este trabajo. La principal de ellas ha sido la fuente de datos utilizada, ya que ésta ha limitado nuestras opciones en cuanto a las variables a incluir en el análisis, y el elevado número de datos que faltaban nos obligó a trabajar con un panel muy incompleto. Además, hay que tener en cuenta que los cambios en las estructuras de propiedad de las empresas como resultado de las fusiones 
producidas han distorsionado alguno de los datos. Debe tenerse presente que de todos los métodos de reestructuración que existen en este análisis solo se tiene en cuenta uno de ellos, la reducción de plantilla llevada a cabo mediante despido del personal lo que ha hecho que se tome la variación de plantilla anual sin saber a ciencia cierta si se trataba de despido o de alguno de los otros métodos de reestructuración.

5. Además, debe tenerse en cuenta otra importante limitación que hemos encontrado en esta investigación, y es que al analizarse únicamente las empresas pertenecientes al sector financiero no se pueden extrapolar íntegramente los resultados obtenidos a todos los sectores y por ende a todas las empresas que operan en nuestro país. Además, existe una limitación temporal en la investigación ya que, al centrarse en una serie temporal de diez años, no tiene un carácter histórico y al estar inmersos aún en una recesión derivada de la crisis no se puede analizar si los efectos de estas reestructuraciones de plantilla son efectivos al no disponer de más años para el análisis.

\section{REFERENCIAS BIBLIOGRÁFICAS}

AHMADJIAN, C. L. y ROBINSON, P. (2001), "Safety in numbers: Downsizing and the deinstitutionalization of permanent employment in Japan", Administrative Science Quarterly, núm 46, 622-654.

ARBOlEDAS, J. R. P., HORTAL, J. P. y DE ENTERRÍA PÉREZ, A. G., (2002), Libro Blanco: Las mejores prácticas (Bestpractices) en los procesos de reestructuración de plantillas, Universidad de Navarra, IESE; CREADE: Sagardoy Abogados.

BAND, D. C. y TUSTIN, C. M. (1995), "Strategic downsizing", Management Decision, núm. 33. 36-45.

BAUMOL, W. J., BLINDER, A. y WOLFF, E. N. (2003), Downsizing in America: Reality, causes, and consequences, New York: Russell Sage Foundation.

BOWMAN, E. H. y SINGH, H. (1993), "Corporate restructuring: Reconfiguring the firm", Strategic Management Journal, núm. 14, 5-14.

BROCKNER, J., GROVER, S., REED, T. F. y DEWITT, R. L. (1992), "Layoffs, job insecurity, and survivors' work effort: Evidence of an invertedU relationship", Academy of Management Journal, núm. 35, 413-425.

BRUTON, G. D., KEELS, J. K. y SHOOK, C. L. (1996), "Downsizing the firm: Answering the strategic questions", The Academy of Management Executive (1993-2005), núm. 10, 38-43.

BUDROS, A. (2002), "The mean and lean firm and downsizing: Causes of involuntary and voluntary downsizing strategies", Sociological Forum, núm. 17, 307-342. 
BUDROS, A. (2000), "Organizational types and organizational innovation: Downsizing among industrial, financial, and utility firms", Sociological Forum, núm. 15, 273-306.

BUDROS, A. (1999), "A conceptual framework for analyzing why organizations downsize", Organization Science, núm. 10, 69-82.

BUDROS, A. (1997), "The new capitalism and organizational rationality: The adoption of downsizing programs, 1979-1994", Social Forces, núm. $76,229-250$.

BUENO, E. (1998), "El capital intangible como clave estratégica en la competencia actual”, Boletín de estudios económicos, núm. 164, 207-229.

CAMERON, K. S. (1994), "Strategies for successful organizational downsizing”, Human Resource Management, 33, núm. 2, 189-211.

CAMERON, K. S., FREEMAN, S. J. y MISHRA, A. K. (1993), "Downsizing and redesigning organizations", Organizational change and redesign, 19-65.

CAPELLI, P. (2000), "Examining the Incidence of Downsizing and its Effect on Establishment Performance”. (Working Paper 7742). National Bureau of Economic Research.

CASCIO, W. F. (1993), "Downsizing: What do we know? What have we learned?", Academy of Management Executive, núm. 7, 95-104.

CASCIO, W. F., YOUNG, C. E. y MORRIS, J. R. (1997), "Financial consequences of employment change decisions in major U.S. corporations", Academy of Management Journal, núm. 40, 1175-1189.

CASILlAS, J. C. y BARROSO, M. C. (1998), "Análisis del efecto de reestructuración en la empresas españolas durante el quinquenio 90-94”, Revista Europea de Dirección y Economía de la Empresa, núm. 7, 53-64.

CLAVER, E. y FERNÁNDEZ, J. A. (2006), "Nuevas perspectivas en los procesos de reestructuración de plantillas", Investigaciones Europeas de Dirección y Economía de la Empresa, núm. 12, 111-131.

COUCKE, K., PENNINGS, E. y SLEUWAEGEN, L. (2007), "Employee layoff under different modes of restructuring: Exit, downsizing or relocation”, Industrial and Corporate Change, núm. 16, 161-182.

DAHL, S. A. y NESHEIM, T. (2010), "Downsizing strategies and institutional environments", Scandinavian Journal Management, núm. 14, 281-348.

DE MEUSE, K., BERGMANN, T., VANDERHEIDEN, P. y RORAFF, C. (2004), "New Evidence Regarding Organizational Downsizing and a Firm's Financial Performance: A Long-term Analysis", Journal of managerial issues, núm. 16, 155-177.

DEWITT, R. L. (1998), "Firm, industry, and strategy influences on choice of downsizing approach”, Strategic Management Journal, núm. 19, 59-79.

DEWITT, R. L. (1993), "The structural consequences of downsizing”, Organization Science, núm.4, 30-40. 
DÍEZ, J., REDONDO, C., BARREIRO, B., y LÓPEZ, M. A. (2002), Administración de empresas. Dirigir en la sociedad del conocimiento, Madrid: Pirámide.

DULWORTH, M. (1991), Evaluación y Mejoramiento de la Productividad, Venezuela: Documentos Clave.

ESPAHBODI, R., JOHN, T., VASUDEVAN, G. (2000), "The Effects of Downsizing on Operating Performance", Review of Quantitative Finance and Accounting, núm. 15, 107-126.

FERNÁNDEZ, J. A. (2006), "¿Qué sabemos realmente acerca de las reestructuraciones de plantillas? Una aportación teórica”, Economic Analysis Working Papers (EAWP), núm.5, 1-17.

FILATOTCHEV, I., BUCK T. y ZHUKOV, V. (2000), "Downsizing in Privatized Firms in Russia, Ukraine, and Belarus", Academy of Management Journal, núm. 43, 286-304.

FREEMAN, S. J. (1994), "Organizational downsizing as convergence or reorientation: implications for human resource management", Human Resource Management, núm. 33, 213-238.

GOMEZ, S. y MARTI, C. (2003), "Las Prejubilaciones y su impacto en la persona, en la empresa y en el sistema de pensiones", IESE Research Papers.

GUTHRIE, J. P. y DATTA, D. K. (2008), "Dumb and dumber: The impact of downsizing on firm performance as moderated by industry conditions", Organization Science, vol. 19, 108-123.

HILLIER, D., MARSHALL, A., MCCOLGAN, P. y WEREMA, S. (2007), "Employee Layoffs, Shareholder Wealth and Firm Performance: Evidence from the UK", Journal of Business Finance $\mathcal{E}$ Accounting, núm. 34, 467-494.

HITT, M. A., HOSKISSON, R. E. y IRELAND, R. D. (1994), “A mid-range theory of the interactive effects of international and product diversification on innovation and performance", Journal of Management, núm.20, 297-326.

HORTAL, P. (2006), "Recolocación o cómo encontrar trabajo rápidamente después de un despido", Capital Humano, núm. 199, 22-28.

HUSELID, M. (1995), "The impact of human resource: Management practices on turnover, Productivity, and corporate Financial performance", Academy of Management Journal, núm. 38, 635-672.

IBORRA, M., DASÍ, Á., DOLZ, C. y FERRER, C. (2007), Fundamentos de dirección de empresas, Madrid: Thomson.

KOONTZ, H., WEIHRICH, H. y CANNICE, M. (2008), Administración. Una perspectiva global y empresarial, México: Mc-Graw Hill.

LOVE, G. y KRAATZ, M. (2009), "Character, conformity, or the bottom line? How and why downsizing affected Corporate reputation", Academy of Management Journal, núm. 52, 314-355. 
LOVE, G. y NOHRIA, N. (2005), "Reducing slack: the performance consequences of downsizing by large industrial firms", Strategic Management Journal, núm. 26, 1087-1108.

MCELROY, J., MORROW, P. y RUDE, S. (2001), “Turnover and organizational performance: a comparative analysis of the effects of voluntary, involuntary and reduction-in-force turnover", Journal of applied Psychology, núm. 86, 1294-1299.

MAGÁN, A. y CÉSPEDES, J. (2007), "Difusión del downsizing en la empresa española. Análisis del modelo de dos etapas", Revista europea de Dirección y Economía de la Empresa, núm. 16, 55-72.

MARKIDES, C. C. (1995), "Diversification, restructuring and economic performance", Strategic Management Journal, núm.16, 101-118.

MARR, R. y GARCÍA, S. G. (1997), La dirección corporativa de los recursos humanos, Madrid: Díaz de Santos.

MARTÍN, D. (2007), "El proceso de reestructuración de plantilla paso a paso", Capital Humano, núm. 206, 68-73.

MELLAHI, K. y WILKILSON, A. (2010), "A Study of the Association between Level of Slack Reduction Following Downsizing and Innovation Output", Journal of Management Studies, núm. 47, 483-508.

MCKINLEY, W., MONE, M. A. y BARKER, V.L. (1998), "Some ideological foundations of organizational downsizing", Journal of Management Inquiry, núm. 7, 198-212.

MENTZER, M. (1996), "Corporate Downsizing and Profitability in Canada”, Canadian Journal of Administrative Sciences, núm.13, 237-250.

MORRIS, J., CASCIO, W. y YOUNG, C. (1999), "Downsizing After all These Years: Questions and Answers About who Did It, How Many Did It, and who Benefited from It", Organizational Dynamics, núm. 27, 78-87.

MUÑOZ, F. y SANCHEZ, M. J. (2011), "Does downsizing improve organisational performance? An analysis of Spanish manufacturing firms", The International Journal of Human Resource Management, núm. 22, 2924-2945.

MUÑOZ, F. y SANCHEZ, M. J. (2010), "Downsizing implementation and financial performance", Management Decision, núm. 48, 1181-1197.

NANTAPORN, M. y KLEINER, B. (2003), "The Effect of Downsizing on Morale y Attrition", Management Research News, núm. 26, 2-4.

NIXON, R. D., HITT, M. A., LEE, H. y JEONG, E. (2004), "Market reactions to announcements of corporate downsizing actions and implementation strategies", Strategic Management Journal, núm.25, 1121-1129.

NONAKA, I. y TAKEUCHI, H. (2007), "The knowledge-creating company", Harvard Business Review, núm. 85, 162. 
ODETTE, M. O., SUÁREZ, I. y VICENTE, J. D. (2009), "Downsizing y su efecto en los resultados en la gran empresa española", Revista europea de Dirección y Economía de la Empresa, núm 18, 13-28.

O'SHAUGHNESSY K. C. y FLANAGAN D. J. (1998), "Determinants of layoff announcements following M\&As: An empirical investigation", Strategic Management Journal, núm. 19, 989-999.

PERRY, T. y SHIVDASANI, A. (2005), "Do boards affect performance? Evidence from corporate restructuring", Journal of Business, núm. 78, 1403. 1431.

PFEIFER, C. (2007), "The Perceived Fairness of Layoffs in Germany: Participation, Compensation, or Avoidance?", Journal of Business Ethics, núm. 74, 25-36.

PICHAULT, F. y RORIVE, B. (2003), “"Restructurations et nouveaux périmètres organisationnels: le rôle de la fonction $\mathrm{RH}$ ”, Actes de la Journée d'études "Les restructurations: volontéoufatalité», GREGOR-IAE de Paris.

REQUEJO, A. (1996), "Reducción de plantilla y problemas de viabilidad financiera: el papel de la estructura de capital", Investigaciones Económicas, núm. 20, 43-70.

ROBBINS, S. P. y COULTER, M. (2005), Administración, México: Prentice Hall.

SANCHEZ, M.J. y SUAREZ, I. (2005), "Los procesos de reducción de plantillas en el ámbito español: motivos y modalidades utilizadas", Revista Europea de Dirección y Economía de la Empresa, núm. 14, 81-96.

SASTRE, M.Á. (1995), "Outplacement. Una aproximación a su realidad teórica.", Cuadernos de Relaciones Laborales, núm. 6, 185.

SHEAFFER, Z., CARMELI, A., STEINER-REVIVO, M. y ZIONIT, S. (2009), "Downsizing strategies and organizational performance: a longitudinal study", Management Decision, núm. 47, 950-974.

SUAREZ, I. (1999), "Decisiones de reducción de la plantilla en la gran empresa española", Investigaciones Europeas de Dirección y Economía de la Empresa, núm. 5, 79-100.

SUAREZ, I. y VICENTE, J. D. (2000), "Decisiones de reestructuración organizativa en la gran empresa española durante la década de los noventa". (Documento de Trabajo 07/00).

WAYHAN, V. B. y WERNER, S. (2000), "The impact of workforce reductions on financial performance: A longitudinal perspective", Journal of Management, núm. 26, 341-363.

WHETTEN, D. A. y GODFREY, P. C. (1998), Identity in organizations: Building theory through conversations, Sage Publications, Inc. 
WORRELL, D. L., DAVIDSON, W. N. y SHARMA. V. M. (1991), "Layoff announcements and stockholder wealth", Academy of Management Journal, núm.43, 662-678.

YOO, H. y MODY, B. (2000), "Predictors of downsizing in the US local telephone industry", Information Society, núm.16, 23-33.

YU, G. y PARK, J. (2006), "The effects of downsizing on the financial performance and employee productivity of Korean Firms", International Journal of Manpower, núm. 27, 230-250. 